\title{
Effects of MiR-375-BMPR2 as a Key Factor Downstream of BMP15/GDF9 on the Smad1/5/8 and Smad2/3 Signaling Pathways
}

\author{
Chang Liu Bao Yuan ${ }^{a}$ Hongyan Chen ${ }^{a}$ Mingqiang Xu Xulei Sun ${ }^{a, b}$ \\ JiaJun Xua,b Yan Gao Chengzhen Chen ${ }^{a}$ Hao Jiang ${ }^{a}$ Jiabao Zhang ${ }^{a}$ \\ aCollege of Animal Sciences, Jilin University, Changchun, 'bShenyang Jiuzhou Family Hospital, Shenyang, \\ P.R. China
}

\section{Key Words}

BMP15/GDF9 • miRNA-375 • BMPR2 • Movine cumulus cells • Smad

\begin{abstract}
Background/Aims: Bone morphogenetic protein 15 (BMP15) and growth differentiation factor 9 (GDF9), which are secreted by oocytes, are important regulators of follicular growth and development and ovarian function. These two factors can regulate the proliferation and apoptosis of cumulus cells via modulation of the Smad signaling pathway. Studies have shown that BMP15 and GDF9 can affect the level of miR-375, whereas the target gene of miR-375 is BMPR2, the type II receptor of BMP15 and GDF9. However, whether or how the BMP15/ GDF9-miR-375-BMPR2 pathway affects the proliferation and apoptosis of bovine cumulus cells through regulation of the Smad signaling pathway remains unclear. Methods: In this study, cumulus cells were first obtained from cumulus-oocyte complexes (COCs). Appropriate concentrations of BMP15 and GDF9 were added during the in vitro culture process. Cell Counting Kit-8 (CCK-8) analyses and flow cytometry were used to determine the effects of BMP15/GDF9 on bovine cumulus cells proliferation and apoptosis. Subsequently, miR-375 mimics, miR-375 inhibitor and BMPR2 siRNA were synthesized and used for transfection experiments. Western Blot analysis was used to detect changes before and after transfection in the expression levels of the BMP15/GDF9 type I receptors ALK4, ALK5 and ALK6; the phosphorylation levels of Smad2/3 and Smad1/5/8, which are key signaling pathway proteins downstream of BMP15/GDF9; the expression levels of PTX3, HAS2 and PTGS2, which are key genes involved in cumulus cells proliferation; and $\mathrm{Bcl} 2 / \mathrm{Bax}$, which are genes involved in apoptosis. Results: The addition of $100 \mathrm{ng} / \mathrm{mL}$ BMP15 or $200 \mathrm{ng} / \mathrm{mL}$ GDF9 or the combined addition of $50 \mathrm{ng} / \mathrm{mL}$ BMP15 and $100 \mathrm{ng} / \mathrm{mL}$ GDF9 effectively inhibited bovine cumulus cell apoptosis and promoted cell proliferation. BMP15/GDF9 negatively regulated miR-375 expression and positively regulated BMPR2 expression. High levels of miR-375 and inhibition
\end{abstract}

Hao Jiang

and Jiabao Zhang

KARGER
College of Animal Sciences, Jilin University,

5333 Xi'an Road, Changchun (P.R. China)

Tel. +8643187836551, E-Mail jhhaojiang@jlu.edu.cn, zjb515@126.com 
of BMPR2 resulted in increased expression of ALK4 and decreased expression of PTX3, HAS2 and PTGS2, whereas miR-375 inhibition resulted in the opposite results. BMP15 and GDF9 significantly activated the levels of $\mathrm{p}-\mathrm{Smad} 2 / 3$ and $\mathrm{p}-\mathrm{Smad} 1 / 5 / 8$, whereas miR-375 inhibited the levels of $p-S m a d 2 / 3$ and $p-S m a d 1 / 5 / 8$ by negatively regulating BMPR2 and also led to apoptosis. Conclusion: BMP15 and GDF9 have synergistic effects and can act through miR375 to affect the expression levels of type I receptor ALK4 and type II receptor BMPR2 and the activation of Smad signaling pathway, which subsequently affected the proliferation, spread and apoptosis of cumulus cells.

\section{Introduction}

Follicles are the basic developmental units in the mammalian ovaries and essentially consist of oocytes and granulosa cells. Granulosa cells can be divided into mural granulosa cells and cumulus cells. Granulosa cells play a decisive role in the development of follicles, and as a type of granulosa cell, cumulus cell proliferation and apoptosis play vital roles in follicular development and oocyte maturation.

Bone morphogenetic protein 15 (BMP15 or GDF9B) and growth differentiation factor-9 (GDF9) are both members of the transforming growth factor (TGF)- $\beta$ superfamily and have similar structures, expression patterns and function and are important regulators of follicular growth and development and ovarian function. BMP15 and GDF9 secreted by oocytes via autocrine and paracrine mechanisms can interact directly (i.e., the formation of heterodimers) or functionally (i.e., playing redundant or antagonistic roles) to regulate local ovarian cell differentiation and the follicle microenvironment, and these proteins promote granulosa cell proliferation and prevent differentiation. Thus, BMP15 and GDF9 play vital roles in follicular development, atresia, ovulation, fertilized egg formation and reproductive maintenance [1-4].

BMP15 can regulate the secretion of some ovarian regulatory factors and the expression of receptors (such as follistatin, KIT ligand, and FSH receptor), thereby promoting the early growth of follicles, controlling the number of dominant follicles before follicle ovulation, and serving as a determinant factor of ovulation quantity and quality. BMP15 can significantly inhibit cumulus cell apoptosis and promote their proliferation by inducing the expression of hyaluronidase 2 (HAS2), pentraxin 3 (PTX3) and prostaglandin synthase 2 (PTGS2) to optimize the oocyte microenvironment and drive oocyte maturation and ovulation [5-8]. GDF9 also has a similar function to that of BMP15 and can inhibit the expression of the LH/ CG receptor (LHCGR) and KIT ligand in cumulus cells, inducing the expression of cumulus cell proliferation-related genes and promoting oocyte maturation and ovulation [9-11].

The reproductive properties of mammals are closely related to BMPs, BMP receptors and Smads, signaling pathway components downstream of the BMP/Smad signaling pathway. In particular, the BMP/Smad signaling pathway plays an irreplaceable role in the regulation of follicular growth and development and granulosa cell growth and differentiation. BMP15 and GDF9 can regulate Smad phosphorylation by binding to activin receptor-like kinase (ALK) family members and then regulating gene transcription by recruiting transcription factors [12], whereas $\operatorname{Smad} 2 / 3$ and $\operatorname{Smad} 1 / 5 / 8$ are key factors for the intracellular signal transduction of the TGF- $\beta$ superfamily of growth factors [13]. The BMP15 and GDF9 signals activate downstream Smad transcription factors via specific complexes formed by binding to type I and type II receptors. The expression of BMP15 type I receptor ALK6 and GDF9 type I receptor ALK5 can be detected in rodent granulosa cells and oocytes; the common type II receptor BMPR2 can also be detected [14], and ALK4 may be the receptor for GDF9 [15]. BMPR2 is essential to the transportation of oocyte signals that act paracrine to cumulus cells and is the only type II receptor for BMP15 and GDF9 [16].

Studies have also shown that miR-375, as an endogenous non-coding RNA, can be used as a proliferation inhibitor and tumor suppressor to inhibit alveolar epithelial cell (AEC) differentiation. At present, it is known that multiple mircoRNAs can regulate the 
expression of the BMPR2 protein, but most previous studies have focused on stem cells or mesenchymal stem cells [17-20]. It is worth mentioning that BMPR2 has been identified as a direct target gene of miR-375, and inhibition of BMPR2 expression has the same inhibitory effect on cell differentiation as does overexpression of miR-375 [21]. Therefore, miR-375 may have a potential role in regulating BMP15- and GDF9-related pathways. However, the specific routes of action and mechanisms are not clear. Our study focused mainly on miR375 and explored how BMP15/GDF9 affects the expression of miR-375, thereby acting on BMPR2 and regulating cumulus cell proliferation and apoptosis through the Smad2/3 and Smad1/5/8 pathways. We hope this study will provide a theoretical basis for the in-depth study of follicular development, oocyte maturation and ovarian function.

\section{Materials and Methods}

\section{Isolation and culture of cumulus cells}

Bovine ovaries were collected from a local halal slaughterhouse and transported to the laboratory in $0.9 \%$ normal saline $\left(37^{\circ} \mathrm{C}\right)$. Follicular fluid was obtained from follicles 3 to $8 \mathrm{~mm}$ in diameter using a 20 - $\mathrm{mL}$ syringe. Under a stereomicroscope, the tightly wrapped cumulus-oocyte complexes (COCs) were dissected and washed 3 times in HEPES (Sigma, St. Louis, MO, USA) buffer. COCs in 0.1\% hyaluronidase (Sigma) were repeatedly pipetted to separate the cumulus cells from the oocytes, and the naked eggs were removed. The remaining cells were collected and resuspended in culture medium containing DMEM/F12 (Gibco, Grand Island, NY, USA), 1\% penicillin and streptomycin (HyClone, Logan, Utah, USA) and 10\% fetal bovine serum (Biological Industries, Kibbutz Beit Haemek, Israel) and cultured in a $38.5^{\circ} \mathrm{C}, 5 \% \mathrm{CO}_{2}$ incubator. Based on specific experimental requirements, recombinant human BMP-15/GDF-9B (R\&D Systems, Minneapolis, MN, USA) and human recombinant GDF9 (Bio Vision, Milpitas, CA, USA) were added when the cells were cultured to the appropriate stages.

\section{Apoptosis detection}

The following steps were performed according to the manual of the Annexin V-FITC Apoptosis Analysis Kit: the cells were washed twice with cold PBS and digested with trypsin without EDTA, after which approximately $1 \times 10^{5}$ cells were collected. The collected cell suspension was centrifuged at $300 \times \mathrm{g} / \mathrm{min}$ for $10 \mathrm{~min}$, and the buffer was removed from the cell pellet. A total of $100 \mu \mathrm{L} 1 \times$ binding buffer was added to each EP tube to resuspend the cells, and $5 \mu \mathrm{L}$ Annexin V-FITC was added to the tube. The tubes were then incubated for $10 \mathrm{~min}$ at room temperature in the dark. Subsequently, $5 \mu \mathrm{L}$ propidium iodide (PI) solution was added, followed by $5 \mathrm{~min}$ of incubation at room temperature. The samples were kept in the dark. The samples for testing were combined with $1 \times$ binding buffer to a total volume of $200 \mu \mathrm{L}$ and used for detection via flow cytometry (BD Biosciences, San Jose, CA, USA) within $1 \mathrm{~h}$ of preparation.

\section{Detection of cell proliferation activity}

The procedures were conducted according to the manual of the CCK-8 (Dojindo, Kumamoto, Japan). The specific steps are as follows. The cell suspension was inoculated into 96-well-plates $(100 \mu \mathrm{L} /$ well, $1 \times 10^{4}$ cells/well). Treatments were applied when the cells grew to approximately $60 \%$ confluency. The treated plates were preincubated in an incubator for $48 \mathrm{~h}$, and $10 \mu \mathrm{L}$ CCK-8 solution was then added to each well. The plates were incubated for $3 \mathrm{~h}$ in an incubator, and the absorbance at $450 \mathrm{~nm}$ was determined using a microplate reader (BioTek Instruments, Winooski, VT, USA).

\section{Transfection of bovine cumulus cells}

RiboFECT $^{\mathrm{TM}}$ CP (Guangzhou RiboBio Co.,Ltd., Guangzhou, China) was used as the cell transfection reagent. NC-mimics RNA (control for the overexpression group), miR-375 mimics (overexpression group), NC-inhibitor RNA (control for the inhibition group), miR-375 inhibitor (inhibition group), NC-siRNA and BMPR2 SiRNA were used for cell transfection. The specific method was as follows: PBS was used to dilute RiboFECT ${ }^{\mathrm{TM}} \mathrm{CP}$ buffer to $1 \times$. A total of $1 \times 10^{5}$ cells were inoculated into 12 -well plates containing complete medium to ensure that the cell density at the time of transfection was $60 \%$. A total of $60 \mu \mathrm{L}$ of $1 \times$ RiboFECT

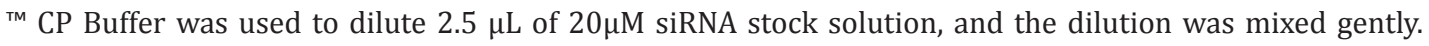




\section{Cellular Physiology Cell Physiol Biochem 2018;46:213-225 \begin{tabular}{l|l} 
and Biochemistry Published onlIne: March 26, 2018 & $\begin{array}{l}\text { (c) } 2018 \text { The Author(s). Published by S. Karger AG, Basel } \\
\text { www.karger.com/cpb }\end{array}$
\end{tabular} \\ Liu et al.: BMP15/GDF9 Regulate Smad1/5/8 and Smad2/3 Signaling through MiR-375- BMPR2}

The solution was added to $6 \mu \mathrm{L}$ of RiboFECT CP Reagent, gently mixed and incubated for 10 min at room temperature. The mixture of RiboFECT ${ }^{\mathrm{T}}$ CP was added to the cell culture medium and gently mixed, and the corresponding concentrations of BMP15 and GDF9 were added to the medium of each group. The plates were incubated in a $5 \% \mathrm{CO}_{2}$ incubator at $38.5^{\circ} \mathrm{C}$ until subsequent experiments. The miRNA-375 mimics/ inhibitor and BMPR2 siRNA were designed and synthesized by Shanghai GenePharma Co., Ltd. The specific sequences are shown in Table 1.

\section{Protein extraction and Western Blot}

More than $1 \times 10^{5}$ cells were collected and lysed in cell lysis buffer (Beyotime, Hangzhou, China) containing $1 \mathrm{mM}$ PMSF for $1 \mathrm{~min}$. At the beginning of cell lysis, the protein phosphatase inhibitor mixture was added at a volume ratio of 99:1. After lysis, the mixture was centrifuged at $13,000 \mathrm{~g} / \mathrm{min}$ for $5 \mathrm{~min}$, and the protein-containing supernatant was collected. After the protein concentration was determined using a Bradford Protein Assay Kit (Beyotime), the protein sample was mixed with SDS-PAGE protein loading buffer (Beyotime) and denatured at $95^{\circ} \mathrm{C}$ for $8 \mathrm{~min}$. Protein samples were separated on a $10 \%$ gel by SDS/ PAGE electrophoresis and were then transferred to a PVDF membrane $(0.22 \mathrm{mM}$, Millipore, MA, USA). The membrane was transferred to $5 \%$ BSA blocking solution and placed on a shaker for 1 $\mathrm{h}$ of blocking. The membrane was then placed in a chamber with primary antibodies diluted to suitable concentrations with the blocking solution and kept on a shaker at $4^{\circ} \mathrm{C}$ overnight, followed by three $5 \mathrm{~min}$ washes with $1 \times$ TBST at room temperature on a shaker. The membrane was then incubated with the corresponding secondary antibody at room temperature for $1 \mathrm{~h}$ followed by three $10 \mathrm{~min}$ washes with $1 \times$ TBST at room temperature on a shaker. Finally, a Tanon 5200 chemiluminescence imaging analyzer (Tanon, Shanghai, China) was used for chromogenesis and imaging. The antibodies information is shown in Table 2. Image software was used for grayscale analysis. All the bands displayed in a single picture may not have originated from the same western blot. However, the target protein and their internal reference are from the same experiment.

\section{Statistical analysis}

SPSS Statistics 20.0 software (IBM Corporation, New York, NY, USA) was used for the statistical analyses. All experimental data are shown as the average value \pm standard error. Differences between different groups of data were tested by the independent-sample t-test or oneway ANOVA. $\mathrm{P}<0.05$ was considered statistically significant, and $\mathrm{P}<0.01$ was considered highly significant.

Table 1. Sequences of miRNA-375 mimics/NC, miRNA-375 inhibitor/NC, siBMPR2 RNA/NC

\begin{tabular}{|c|c|}
\hline Name & Sequence( $5^{\prime}$ to $3^{\prime}$ ) \\
\hline \multirow{2}{*}{ iR-375 mimics } & JUGUUCGUUCGGCUCGCG \\
\hline & CGCGAGCO \\
\hline \multirow{2}{*}{ miR-375 mimics NC } & sense: UUCUCCGAACGUGUCACGUT \\
\hline & antisense: ACGUGACACGUUCGGAGA \\
\hline \multirow{4}{*}{$\begin{array}{l}\text { miR-375 inhibitor } \\
\text { miR-375 inhibitor NC } \\
\text { BMPR2-bos-1703 }\end{array}$} & sense: UCACGCGAGCCGAACGAACAA \\
\hline & sense: CAGUACUUUUGUGUAGUACAP \\
\hline & sense: GCUUGUGAUGGAGUAUUAI \\
\hline & antisense: AUAAUACUCCAUCACAAG \\
\hline \multirow{2}{*}{ BMPR2-bos-1703 NC } & Inse: UUCUCCGAACGUGUCACGUTT \\
\hline & e: ACGUGACACGUUCGGAGA \\
\hline
\end{tabular}

Table 2. Antibodies used for western blot

\begin{tabular}{ll}
\hline Antibody name & Company, catalog number \\
\hline BMPR2 & CST, \#6979 \\
ALK4 & Abcam, ab109300 \\
ALK5 & Santa Cruz, sc-398 \\
ALK6 & Abcam, ab155058 \\
ALK7 & Abcam, ab71539 \\
Smad2/3 & CST, \#3102 \\
Phospho-Smad2/3 & CST, \#8828 \\
Smad1/5/8 & Abcam, ab66737 \\
Phospho-Smad1/5/8 & CST, \#13820 \\
PTGS2 & CST, \#12282 \\
HAS2 & Abcam, ab131364 \\
PTX3 & Abcam, ab94649 \\
Bcl2 & Abcam, ab183656 \\
Bax & Abcam, ab32503 \\
GAPDH & CST, \#2118 \\
\hline
\end{tabular}




\section{Results}

Effects of different concentrations of BMP15 and GDF9 on apoptosis and proliferation of bovine cumulus cells

Flow cytometry and CCK-8 testing showed that the proliferation (Fig. 1A) and apoptosis (Fig. 1B) of cumulus cells relied on BMP15 and GDF9 concentrations in a dose-dependent manner. Cumulus cell proliferation gradually increased and apoptosis gradually decreased with increasing BMP15 concentrations up to $100 \mathrm{ng} / \mathrm{mL}$. At a concentration of $200 \mathrm{ng} / \mathrm{mL}$, the cell proliferation ability was greatly reduced, and the apoptotic rate increased significantly. For GDF9, cumulus cell proliferation increased gradually and the apoptosis rate decreased gradually with increasing concentrations up to $200 \mathrm{ng} / \mathrm{mL}$; at a concentration of $300 \mathrm{ng} / \mathrm{mL}$, the cell proliferation ability was significantly reduced and the apoptosis rate increased significantly. The effects of combined BMP15 and GDF9 treatment on cumulus cell apoptosis and proliferation were similar to those observed with single-agent treatment. When the concentration of BMP15 was $50 \mathrm{ng} / \mathrm{mL}$ and the concentration of GDF9 was $100 \mathrm{ng} / \mathrm{mL}$, the cumulus cell apoptosis rate was the lowest and the cell proliferation ability was highest. Therefore, we selected the concentrations of 100 ng/mL BMP15, $200 \mathrm{ng} / \mathrm{mL}$ GDF9, and $50 \mathrm{ng} /$ mL BMP15 + $100 \mathrm{ng} / \mathrm{mL}$ GDF9 for subsequent studies.

\section{Effects of BMP15 and GDF9 on the expression} of $\mathrm{miR}-375$ and BMPR2

After supplementation with BMP15, GDF9 and BMP15/GDF9, the changes in the expression levels of miR-375 and BMPR2 in cumulus cells were detected as shown in Fig. 2. For each type of supplementation, the expression level of miR-375 was significantly decreased (Fig. 2A), but miR-375 expression was not significantly different among the three types of supplementation. On the whole, BMPR2 expression increased significantly in the BMP15, GDF9 and combined supplementation groups, but there were significant differences among the groups. The combination group had the highest level of BMPR2 expression, followed by the GDF9 group and then the BMP15 group (Fig. 2B).

Effects of miR-375 and BMPR2 on the expression levels of ALK4/5/6 as key regulators downstream of BMP15 and GDF9

The miR-375 mimics, miR-375 inhibitor and BMPR2-siRNA were added following BMP15, GDF9 and BMP15/GDF9 addition. The expression of ALK4 in the miR-375 mimics

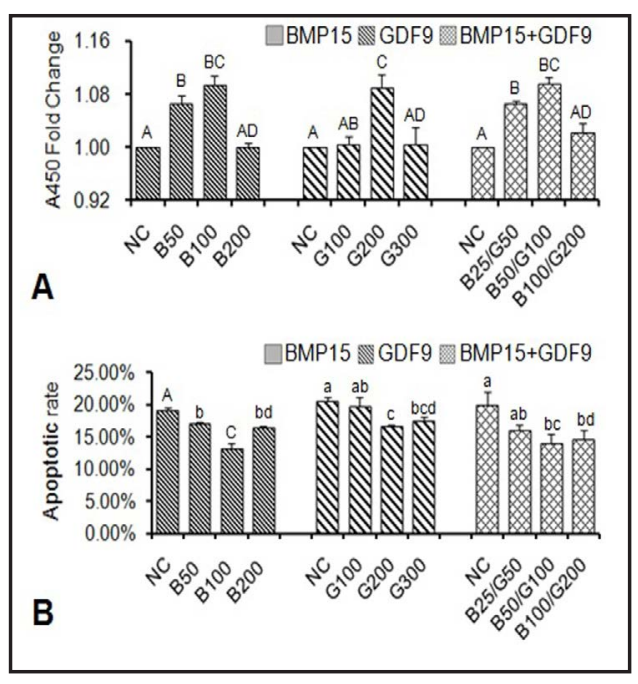

Fig. 1. Effects of different concentrations of BMP15, GDF9 and BMP15/GDF9 on the proliferation and apoptosis of bovine cumulus cells. B50, B100 and B200 indicate that the added concentrations of BMP15 were $50 \mathrm{ng} /$ $\mathrm{mL}, 100 \mathrm{ng} / \mathrm{mL}$, and $200 \mathrm{ng} / \mathrm{mL}$, respectively; G100, G200 and G300 indicate that the added concentrations of GDF9 were $100 \mathrm{ng} / \mathrm{mL}, 200$ $\mathrm{ng} / \mathrm{mL}$, and $300 \mathrm{ng} / \mathrm{mL}$, respectively; and B25/ G50, B50/G100, and B100/G200 indicate that in combined additions, the BMP15 concentrations were $25 \mathrm{ng} / \mathrm{mL}, 50 \mathrm{ng} / \mathrm{mL}$, and $100 \mathrm{ng} /$ $\mathrm{mL}$ and the GDF9 concentrations were $50 \mathrm{ng} /$ $\mathrm{mL}, 100 \mathrm{ng} / \mathrm{mL}$, and $200 \mathrm{ng} / \mathrm{mL}$, respectively. NC represents the corresponding control groups in which only the solvents of BMP15 and GDF9 were added. (A) The proliferation of cumulus cells relied on BMP15 and GDF9 in a dose-dependent manner. (B) The apoptotic rate of cumulus cells relied on BMP15 and GDF9 in a dose-dependent manner. In the same group, numbers labeled with different letters indicate significant differences $(\mathrm{P}<0.05)$. Different letters of different cases indicate highly significant differences $(\mathrm{P}<0.01)$. Numbers labeled with the same letters indicate that the difference was not significant $(\mathrm{P}>0.05)$. 
Fig. 2. Effects of BMP15 and GDF9 on the expression of miR-375 and BMPR2. Mean values labeled with different letters indicate significant differences $(\mathrm{P}<0.05)$, and those labeled with different letters of different cases indicate that the difference was highly significant $(\mathrm{P}<0.01)$. (A) Relative expression changes of miRNA-375 after BMP15, GDF9 or BMP15/ GDF9 addition. In contrast to the NC group, the expression levels of miR-375 after the addition

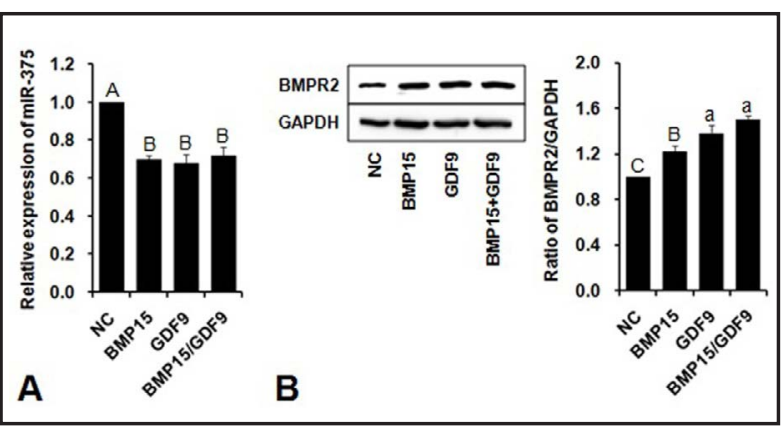
of BMP15 or GDF9 decreased by more than

$25 \%$ compared with that before supplementation. (B) Relative expression changes of BMPR2 after BMP15, GDF9 or BMP15/GDF9 addition. The BMPR2 band was approximately $150 \mathrm{kDa}$, and the GAPDH band was approximately $37 \mathrm{kDa}$. BMPR2 expression increased by approximately $22 \%$ in the BMP 15 group, approximately $38 \%$ in the GDF9 group, and approximately $51 \%$ in the BMP15/GDF9 combination group.

group was significantly increased (Fig. 3A). In the miR-375 inhibitor group, the expression level of ALK4 was significantly decreased (Fig. 3B). In the BMPR2 siRNA group, the expression level of ALK4 was significantly increased (Fig. 3C). The expression levels of ALK5 and ALK6 did not change significantly with any of the treatments.

\section{Effects of miR-375 and BMPR2 on the phosphorylation levels of Smad1/5/8 and Smad2/3 as key regulators downstream of BMP15/GDF9}

The application of miR-375 mimics, miR-375 inhibitor and BMPR2 siRNA following supplementation with BMP15, GDF9 and BMP15+GDF9 had no significant effect on the expression levels of Smad1/5/8 or Smad2/3. The addition of miR-375 mimics and BMPR2 siRNA alone significantly reduced the levels of $\mathrm{p}$-Smad1/5/8 and $\mathrm{p}$-Smad2/3, which was significantly alleviated by supplementation with BMP15, GDF9 or BMP15/

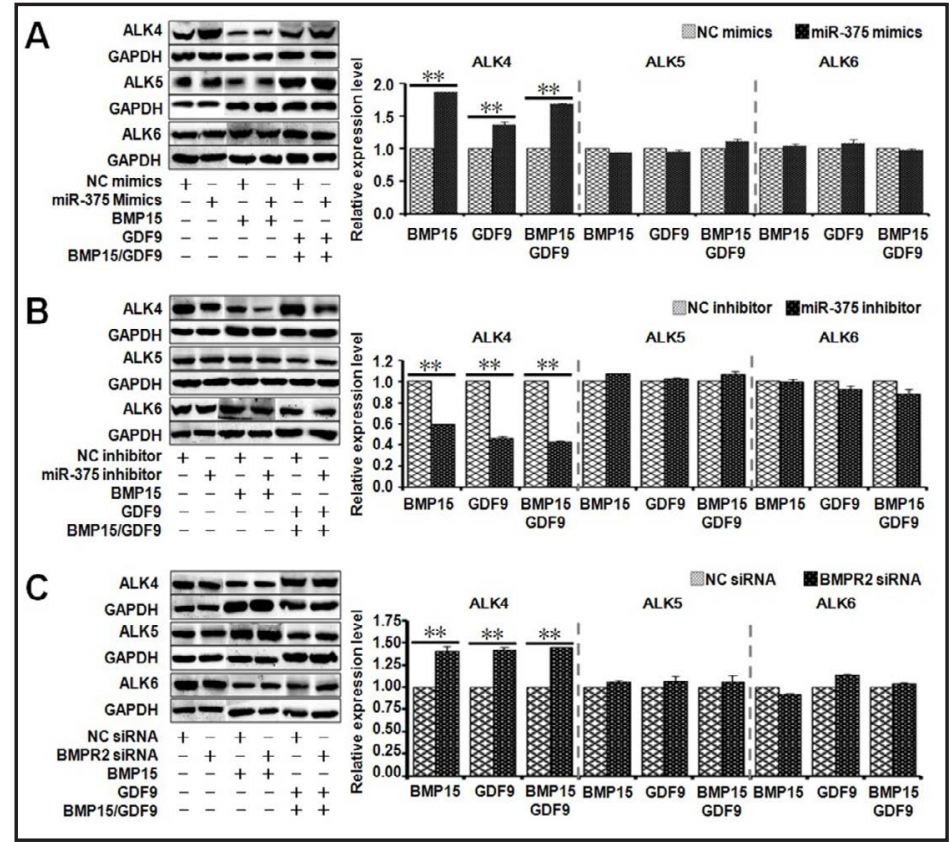

Fig. 3. Effect of miR-375 and BMPR2 on the expression of ALK4/5/6 as key regulators downstream of BMP15 and GDF9. ** indicates that the difference was highly significant $(\mathrm{P}<0.01)$. The ALK4 band was approximately $57 \mathrm{kDa}$, the ALK5 band was approximately $53 \mathrm{kDa}$, the ALK6 band was approximately $57 \mathrm{kDa}$, and the GAPDH band was $37 \mathrm{kDa}$. "+"means with addition, "-" means without addition. ALK5 and ALK6 showed no significant changes following the BMP15 or GDF9 treatment. (A) Relative expression changes of ALK4/5/6 after miR-375 mimics addition. Overexpression of miR-375 increased the expression of ALK4 by $86 \%$, $36 \%$ and 83\%in the BMP15, GDF9 and combined addition groups, respectively. (B) Relative expression changes of ALK4/5/6 after miR375 inhibitor addition. Inhibition of miR-375 decreased ALK4 by 57\%, $54 \%$ and 41\%in the BMP15, GDF9 and combined addition groups, respectively. (C) Relative expression changes of ALK4/5/6 after BMPR2siRNA addition. Inhibition of BMPR2 increased the expression of ALK4 by $40 \%, 42 \%$ and $44 \%$ in the BMP15, GDF9 and combined addition groups, respectively.

\section{KARGER}


Fig. 4. Effects of miR-375 and BMPR2 on Smad1/5/8 signaling pathway as the key regulators downstream of BMP15 and GDF9. ** indicates that the difference was highly significant $(\mathrm{P}<0.01)$. The Smad $1 / 5 / 8$ bands were approximately $62 \mathrm{kDa}$ and $52 \mathrm{kDa}$, and the GAPDH band was $37 \mathrm{kDa}$. (A) Relative expression changes of $\operatorname{Smad} 1 / 5 / 8$ and $\mathrm{p}$-Smad1/5/8 after miR-375 mimics addition. The addition of the miR-375 mimic reduced the level of p-Smad $1 / 5 / 8$ by more than $85 \%$, and the addition of BMP15, GDF9 or BMP15/GDF9 resulted in the recovery of p-Smad $1 / 5 / 8$ levels to approximately $36 \%, 60 \%$ or $81 \%$ that of the

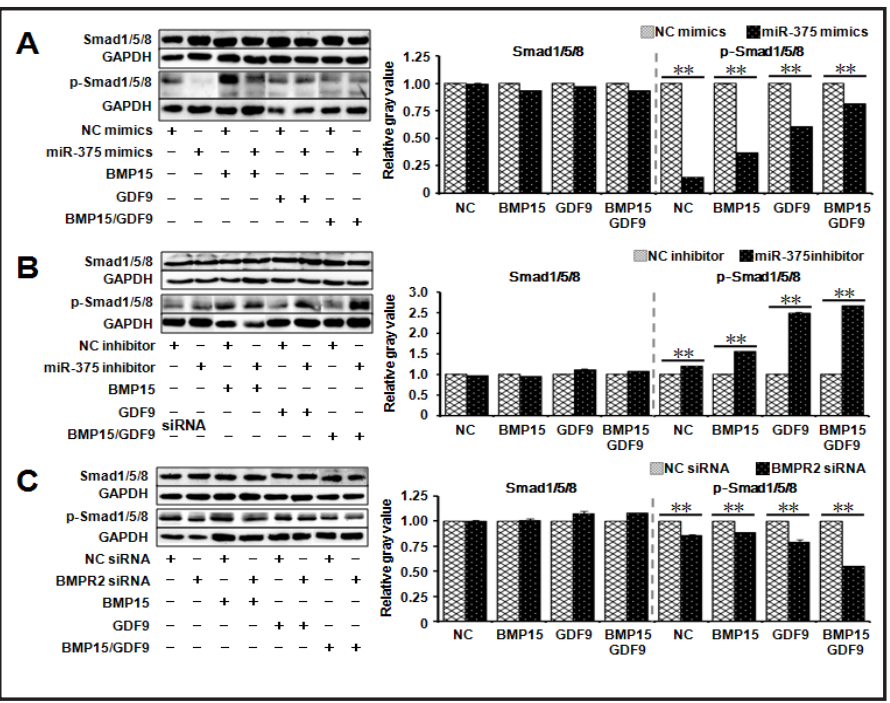
control group, respectively. (B) Relative expression changes of Smad1/5/8 and p-Smad1/5/8 after miR-375 inhibitor addition. The addition of the 375 inhibitor increased the p-Smad1/5/8 level by 19\%, whereas the further addition of BMP15, GDF9 or BMP15/GDF9 increased the level of p-Smad1/5/8 by $56 \%, 150 \%$ or $166 \%$, respectively. (C) Relative expression changes of Smad1/5/8 and p-Smad1/5/8 after BMPR2 siRNA addition. The results of the addition of BMPR2-siRNA were similar to those observed with the addition of the miR-375 mimic, which significantly reduced p-Smad1/5/8, and the addition of BMP15, GDF9 or combined agents reduced p-Smad1/5/8 by $12 \%, 21 \%$ or $45 \%$, respectively.

Fig. 5. Effects of miR-375 and BMPR2 on the Smad2/3 signaling pathway as key regulators downstream of BMP15 and GDF9. * Indicates a significant difference within the same group $(\mathrm{P}<0.05)$, ** indicates a highly significant difference within the same group $(\mathrm{P}<0.01)$. The $\operatorname{Smad} 2 / 3$ bands were approximately $60 \mathrm{kDa}$ and $52 \mathrm{kDa}$, and the GAPDH band was $37 \mathrm{kDa}$. (A) Relative expression changes of $\operatorname{Smad} 2 / 3$ and $\mathrm{p}-\operatorname{Smad} 2 / 3$ after miR-375 mimics addition. The addition of the miR-375 mimic significantly inhibited the level of $\mathrm{p}$-Smad2/3, and even with the addi-

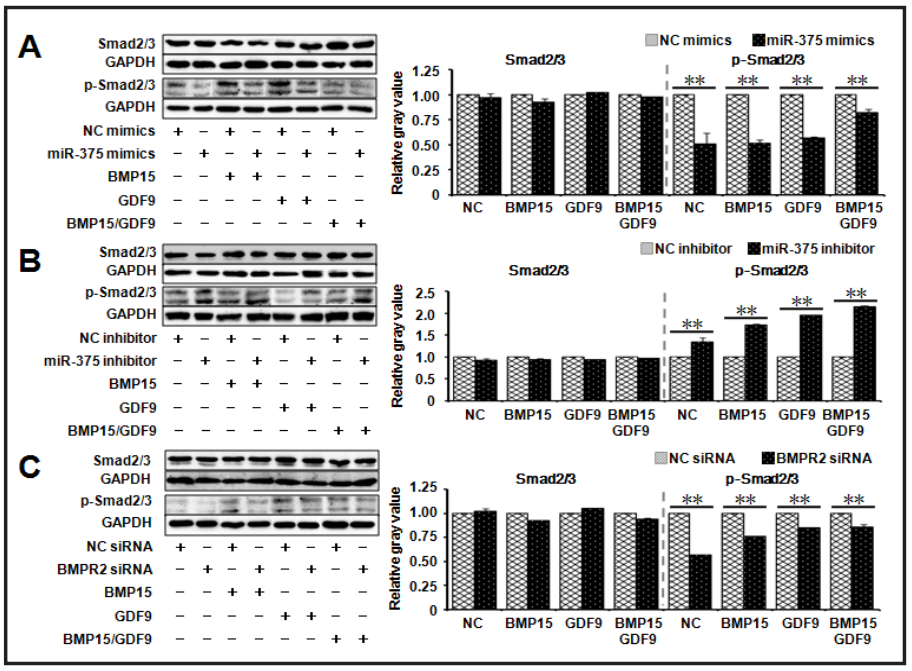
tion of BMP15 or GDF9, the level of p-Smad2/3 was still close to that of the group with no addition of BMP or GDF9 and still decreased by nearly 50\%. Although the combined addition of BMP15/GDF9 caused the p-Smad2/3 level to recover to nearly $81 \%$, the level was still lower than that of the control group. (B) Relative expression changes of Smad2/3 and p-Smad2/3 after miR-375 inhibitor addition. The addition of the miR-375 inhibitor increased p-Smad $2 / 3$ by 33\%, the additional supplementation of BMP15 or GDF9 increased p-Smad $2 / 3$ by $72 \%$ or $95 \%$, respectively, and the combined supplementation of BMP15/GDF9 increased p-Smad2/3 by $115 \%$. (C) Relative expression changes of Smad2/3 and p-Smad2/3 after BMPR2 siRNA addition. The effect of adding BMPR2-siRNA was similar to that of the miR-375 mimic, which significantly reduced p-Smad2/3. With the addition of BMP15, GDF9 or BMP15/GDF9, p-Smad2/3 recovered to $76 \%, 85 \%$ or $86 \%$ of that of the control group, respectively, but was still lower than in the control group. 
Liu et al.: BMP15/GDF9 Regulate Smad1/5/8 and Smad2/3 Signaling through MiR-375BMPR2

Fig. 6. Expression changes of PTX3, HAS2 and PTGS2. ** indicates a highly significant difference $(\mathrm{P}<0.01)$. For PTX3, the addition of miR-375 mimics alone decreased the protein level to approximately $65 \%$, and simultaneous addition of the miR-375 mimics and BMP15, GDF9 or BMP15/GDF9 increased the protein levels by $56 \%, 51 \%$ and $93 \%$, respectively. The addition of the miR-375 inhibitor alone increased the protein level by approximately $22 \%$, and simultaneous addition of BMP15, GDF9 or BMP15/GDF9 on top of the miR-375 inhibitor increased the protein level by $50 \%, 24 \%$ and $154 \%$, respectively. The results of BMPR2-siRNA alone were similar to those of the miR-375 mimics alone, which reduced PTX3 by 19\%. Simultaneous addition of BMP15, GDF9 or BMP15/GDF9 on top of

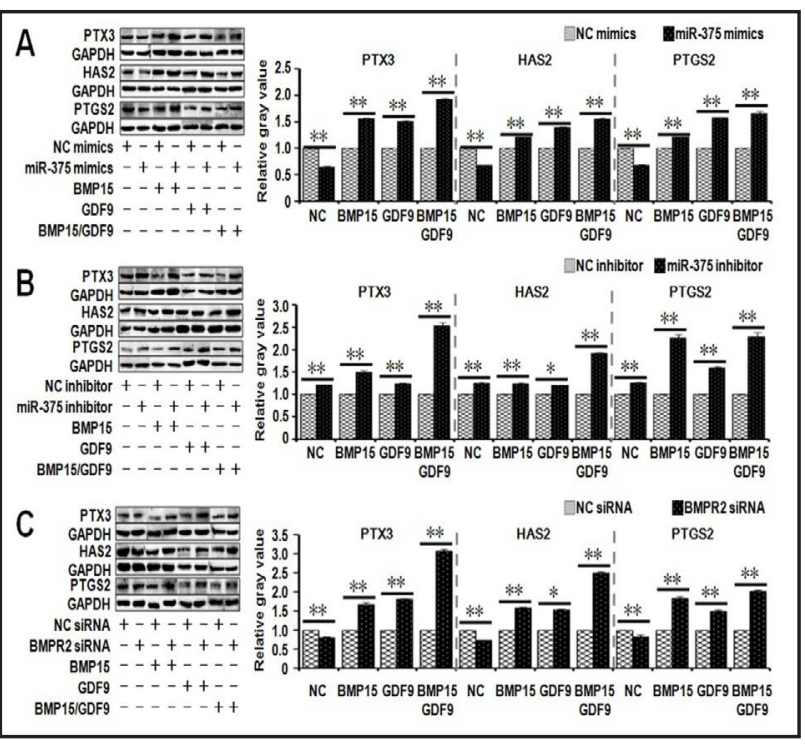
BMPR2-siRNA increased the protein level by $68 \%, 81 \%$ and $207 \%$, respectively. For HAS2, the addition of miR-375 mimics decreased the protein level by $32 \%$, and the simultaneous addition of BMP15, GDF9 or BMP15/GDF9 on top of the miR-375 mimics increased the protein level by $21 \%, 40 \%$ or $55 \%$, respectively. The addition of the miR-375 inhibitor alone increased the protein expression level by approximately $25 \%$, and the simultaneous addition of BMP15, GDF9 or BMP15/GDF9 on top of the miR-375 inhibitor increased the protein level by $24 \%, 20 \%$ or $93 \%$, respectively. The results of the BMPR2 siRNA-alone treatment were similar to those of miR-375 mimics alone, leading to a $26 \%$ decrease in the HAS2 level. Simultaneous addition of BMP15, GDF9 or BMP15/GDF9 on top of BMPR2 siRNA increased the protein level by $59 \%, 54 \%$ or $150 \%$, respectively. For PTGS2, the addition of miR-375 mimics alone decreased the protein level by $32 \%$, whereas the simultaneous addition of BMP15, GDF9 or BMP15/GDF9 on top of the miR-375 mimics increased the protein level by $22 \%, 58 \%$ or $66 \%$, respectively. The addition of the miR-375 inhibitor alone increased the protein level by approximately $25 \%$, whereas the simultaneous addition of BMP15, GDF9 or BMP15/GDF9 on top of the miR-375 inhibitor increased the protein level by $126 \%, 60 \%$ or $130 \%$, respectively. The addition of BMPR 2 siRNA alone was similar to that of the miR-375 mimics alone, which decreased the PTGS2 level by $16 \%$. Simultaneous addition of BMP15, GDF9 or BMP15/GDF9 on top of BMPR2 siRNA increased the protein level by $83 \%, 50 \%$ or $102 \%$, respectively.

GDF9 (Fig. 4 and Fig. 5). However, the level of p-Smad1/5/8 was still highly significantly lower than that of the mimics-NC group. The addition of miR-375 inhibitor alone significantly increased the levels of $\mathrm{p}-\mathrm{Smad} 1 / 5 / 8$ and $\mathrm{p}-\mathrm{Smad} 2 / 3$, whereas the addition of BMP15, GDF9 and BMP15/GDF9 on top of the miR-375 inhibitor further enhanced the expression of $\mathrm{p}-\mathrm{Smad} 1 / 5 / 8$ and $\mathrm{p}-\mathrm{Smad} 2 / 3$.

Effects of miR-375 and BMPR2 on the expression of the cumulus cell proliferation genes, PTX3, HAS2 and PTGS2

The addition of the miR-375 mimics highly significantly reduced the protein expression levels of PTX3, HAS2 and PTGS2. However, the protein expression levels of PTX3, HAS2 and PTGS2 were highly significantly increased after the simultaneous addition of miR-375 mimics with BMP15, GDF9 and BMP15/GDF9 (Fig. 6A). After the addition of the miR-375 inhibitor alone, the expression levels of PTX3, HAS2 and PTGS2 were highly significantly increased. When the miR-375 inhibitor was added together with the addition of BMP15, GDF9 and BMP15/GDF9, the levels of PTX3, HAS2 and PTGS2 were higher than when the miR-375 inhibitor was added alone (Fig. 6B). The expression changes in the PTX3, HAS2 and PTGS2 proteins in the BMPR2 siRNA-alone group were similar to those in the miR-375 mimics group, and all proteins showed highly significantly decreased expression levels. After 
Liu et al.: BMP15/GDF9 Regulate Smad1/5/8 and Smad2/3 Signaling through MiR-375BMPR2

Fig. 7. Effects of BMP15 and GDF9 on the apoptosis of cumulus cells by regulating miR-375 and BMPR2. * Indicates a significant difference, ${ }^{* *}$ indicates a highly significant difference. When the miR-375 mimics were added on top of BMP15, GDF9 or BMP15/GDF9 supplementation, the ratio of $\mathrm{Bcl} 2$ to $\mathrm{Bax}$ was decreased by $48 \%, 66 \%$ or $79 \%$, respectively, and the corresponding apoptotic rate was increased from $8.93 \%, 7.86 \%$ or $7.48 \%$ to $11.16 \%, 10.92 \%$ or $10.58 \%$, respectively. While the other conditions remained unchanged, the $\mathrm{Bcl} 2$ : Bax ratio and apoptosis rate did not change significantly before vs. after the addition of

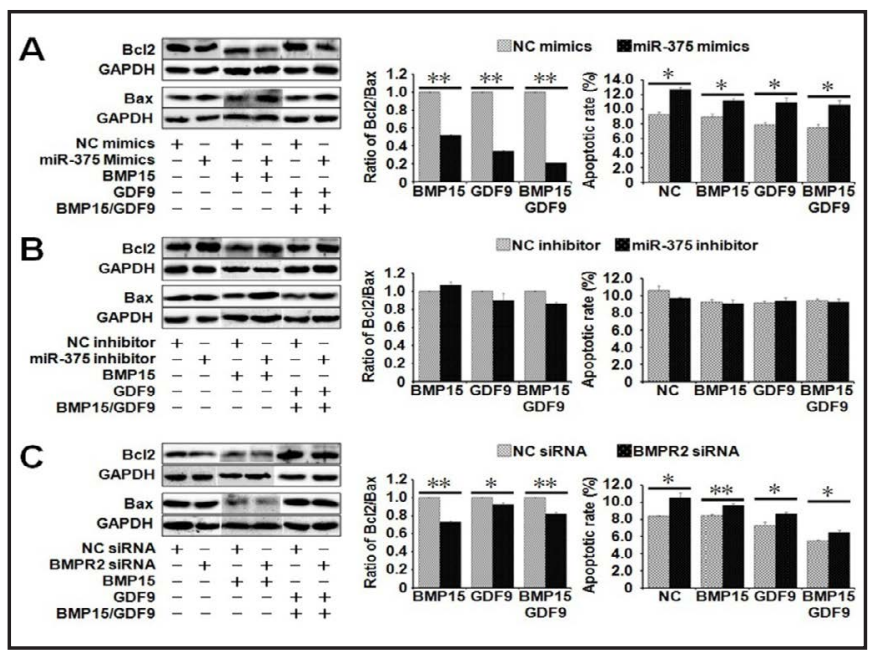
inhibitor. After the addition of BMPR2-

siRNA, the ratio of Bcl2 to Bax was similar to that after the addition of the miR-375 mimics. The ratio of Bcl2 to Bax decreased by 27\%, 7.9\% and 18\%in the BMP15 group, GDF9 group and BMP15/GDF9 group, respectively, and the corresponding apoptotic rates increased from $8.46 \%, 7.28 \%$ and $5.51 \%$ to $9.64 \%, 8.65 \%$ and $6.49 \%$, respectively.

the addition of BMP15, GDF9 and BMP15/GDF9, the protein levels of PTX3, HAS2 and PTGS2 were also significant increased compared with the corresponding control group (Fig. 6C).

Effects of BMP15 and GDF9 on the apoptosis of cumulus cells by regulating miR-375 and BMPR2

When the miR-375 mimics were added together with BMP15, GDF9 or BMP15/GDF9, the ratio of $\mathrm{Bcl} 2$ : Bax was significantly decreased, with the greatest reduction observed in the BMP15/GDF9 group. We used flow cytometry to detect cumulus cell apoptosis, and the results showed that the apoptotic rates were significantly increased after adding the miR-375 mimics, no matter whether BMP15, GDF9 and BMP15/GDF9 were added. When the miR-375 inhibitor was added together with BMP15, GDF9 or BMP15/GDF9, the ratio of $\mathrm{Bcl} 2$ to Bax was not significantly changed, and cumulus cell apoptosis was not significantly affected. No matter whether BMP15, GDF9 or BMP15/GDF9 were added, the ratio of Bcl2 to Bax in the groups with BMPR2-siRNA was significantly lower than in the control group, and the apoptosis rate of bovine cumulus cells was significantly increased.

\section{Discussion}

Oocytes are the core components of mammalian follicles and can secrete oocyte secretion factors (OSFs), such as GDF9 and BMP15, to activate the Smad signaling pathway and thereby extensively regulate granulosa cell and cumulus cell functions, including differentiation, proliferation, apoptosis and luteinization [22]. These actions subsequently affect mammalian reproductive capacity. Recent studies have shown that one of the target genes of miR-375 is BMPR2, the common and only type II receptor of BMP15 and GDF9 [21, 23]. However, whether miR-375 affects other receptors of BMP15 and GDF9 in the process and whether miR-375 regulates the activation of the Smad signaling pathway by regulating BMPR2 remain unclear.

BMP15 and GDF9 are both cytokines that are specifically secreted by oocytes. To clarify the roles of miR-375 and BMPR2 in the BMP15 and GDF9-mediated regulation of cumulus cells, we first added different concentrations of BMP15 and GDF9 to treat cumulus cells in vitro. The results showed that BMP15, GDF9 and BMP15/GDF9 promoted cumulus cell 
proliferation and prevented apoptosis within a certain concentration range, but the effects of BMP15 and GDF9 were not obvious, and BMP15 and GDF9 may have even inhibited cell proliferation and triggered apoptosis when the concentrations were too high (Fig. 1), which may be related to cell tolerance and the toxicity of impurities. Wu et al. reported that miR375 significantly inhibited OSCC cell viability, proliferation, invasion and migration and induced apoptosis [24].

The BMP15 and GDF9 common type II receptor BMPR2 is the target gene for both BMP15 and GDF9. From the results of this study, it can be seen that the addition of BMP15 or GDF9 caused miR-375 down-regulation and BMPR2 up-regulation, and similar to some previous reports, the combined addition of BMP15/GDF9 showed some synergistic effects [13]. This result suggested that oocytes may affect the expression of endogenous miR-375 in surrounding cumulus cells by regulating the secretion of BMP15 and GDF9 and subsequently regulating the expression of BMPR2, leading to cumulus cell proliferation or apoptosis. MiR375 has a key restriction role during the process by which oocytes secrete BMP15 and GDF9 to regulate the physiological state of surrounding cumulus cells.

In addition to type II receptors, BMP15 and GDF9 bind to their respective type I receptors (ALK4, ALK5 and ALK6) to regulate follicular growth and granulosa cell growth and differentiation through the Smad signaling pathway. The results of this study showed that miR-375 can indirectly regulate the expression of ALK4 via its target gene BMPR2 but has no effect on ALK5/6. On the other hand, ALK4 itself is a pro-apoptotic factor $[25,26]$. The addition of miR-375 also upregulates ALK4, which is consistent with the function of miRNA-375 itself in promoting apoptosis. Treatments with BMP15, GDF9 and BMP15/GDF9 plusmiR-375 mimics, miR-375 inhibitor or BMPR2-siRNA showed regulatory effects on ALK4. Therefore, in addition to its effect on the type II receptor BMPR2, miR-375 could also regulate the type I receptor ALK4 of BMP15 and GDF9 via its effects on BMPR2.

BMP15 and GDF9 can regulate the proliferation, differentiation and migration of cells by activating Smad1/5/8 and Smad2/3 [27, 28]. In our study, BMP15, GDF9, BMP15/GDF9, miR-375 and BMPR2 had no significant effect on the level of Smad1/5/8 orSmad2/3 but had varying degrees of influence on p-Smad1/5/8 and p-Smad2/3. MiR-375 is a key factor downstream of BMP15 and GDF9 and can act through its target gene BMPR2 to exert a significant negative effect on $\mathrm{p}-\mathrm{Smad} 1 / 5 / 8$ and $\mathrm{p}-\mathrm{Smad} 2 / 3$. Overexpression of miR-375 reduced $p-S m a d 1 / 5 / 8$ and $p-S m a d 2 / 3$ by more than half, whereas addition of BMP15, GDF9 or BMP15/GDF9 alleviated this reduction (Fig. 4A and Fig. 5A). In contrast, inhibition of miR-375 expression significantly increased the levels of p-Smad1/5/8 and p-Smad2/3, whereas addition of BMP15, GDF9 and BMP15/GDF9 further increased the levels of p-Smad1/5/8 and p-Smad2/3 on top of miR-375 inhibition (Fig. 4B and Fig. 5B). These results indicated that the GDF9 and BMP15 activation of Smad1/5/8 and Smad2/3 may involve multiple pathways, but miR-375/BMPR2 is one of the key pathways. In terms of the added concentrations, the concentrations of BMP15 and GDF9 in BMP15/GDF9 were both half of the concentrations used for the individual treatment of BMP15 and GDF9. However, the enhancement of $\mathrm{p}-\mathrm{Smad} 1 / 5 / 8$ and $\mathrm{p}-\mathrm{Smad} 2 / 3$ levels caused by the combined treatment of BMP15/GDF9 was significantly higher than that due to GDF9 or BMP15 alone. This finding suggested that BMP15/GDF9 has a pronounced synergistic effect on the p-Smad1/5/8 and p-Smad2/3 pathways.

In the in vitro COCs culture experiments, cumulus cell proliferation and expansion had important effects on oocyte maturation and quality, whereas PTX3, HAS2 and PTGS2 are important genes that affected cumulus cell proliferation [29, 30].This study showed that miR-375 could regulate the expression levels of PTX3, HAS2 and PTGS2 through its target genes. Overexpression of miRNA-375 and knockdown of BMPR2 both inhibited the expression of PTX3, HAS2 and PTGS2, whereas the addition of inhibitor led to the opposite result. There might be several reasons for this observation. First, bioinformatics predictions suggested that PTX3, HAS2 and PTGS2 are not the target genes of miR-375, and miR-375/ BMPR2 does not directly regulate PTX3, HAS2, and PTGS2. The reason for decreased levels of PTX3, HAS2 and PTGS2 may be because miR-375 triggers a large number of cells to undergo 
apoptosis, which is also supported by some previous reports [17, 19, 25] and our followup experiments (Fig. 7). Second, under normal physiological conditions, although miR-375/ BMPR2 has a regulatory effect on PTX3, HAS2, and PTGS2, this effect is dependent on BMP15 and GDF9 or is dominated by BMP15 and GDF9. In the in vitro culture experiments, additional treatment with BMP15 and GDF9 significantly alleviated the inhibition of miR-375/BMPR2 on PTX3, HAS2 and PTGS2, suggesting that BMP15 and GDF9 have a more effective pathway for the regulation of cumulus cell proliferation. In addition, in terms of the cumulus cell proliferation genes, we found that the expression of PTX3, HAS2 and PTGS2 was the highest after the combined addition of BMP15 and GDF9, compared with the individual treatments. These results suggested that BMP15/GDF9 have significant synergistic effects on cumulus cell proliferation.

Through the detection of the expression levels of apoptosis-related genes, we found that the Bcl2: Bax ratio was significantly reduced and the proportion of cumulus cell apoptosis was increased after the over-expression of miR-375 or inhibition of BMPR2. This could be the main reason leading to downregulated expression of genes such as PTX3, thus affecting the expansion of cumulus cells. We also observed that the addition of BMP15 and GDF9 did not prevent apoptosis caused by miR-375 mimics but had a mitigating effect on apoptosis caused by inhibition of BMPR2, suggesting that miR-375 and BMPR2 are both key cell apoptosis regulators downstream of BMP15 and GDF9. In contrast, after the inhibition of miR-375, the Bcl2: Bax ratio did not change significantly, and cells did not show significant apoptosis. This may be because under the normal cell culture system conditions, the cells have a very low apoptosis rate, and removal of pro-apoptotic factors will not lead to obvious changes.

\section{Conclusion}

In summary, this study explored the function of miR-375 and BMPR2 as downstream regulators of BMP15 and GDF9 and found that BMP15 and GDF9 have significant synergistic effects in activating the Smad signaling pathway and cumulus cell proliferation (Fig. 8). Our study provides a theoretical basis for gaining an in-depth understanding of the molecular regulation mechanism of follicular development and oocyte maturation and provides theoretical support for the establishment of a more comprehensive oocyte in vitro maturation system and for the full evaluation of animal egg resources. However, BMP15 and GDF9 are known to play extensive roles, the target gene of the downstream miR375 may not be unique, and other key regulatory factors of this pathway need to be further investigated.

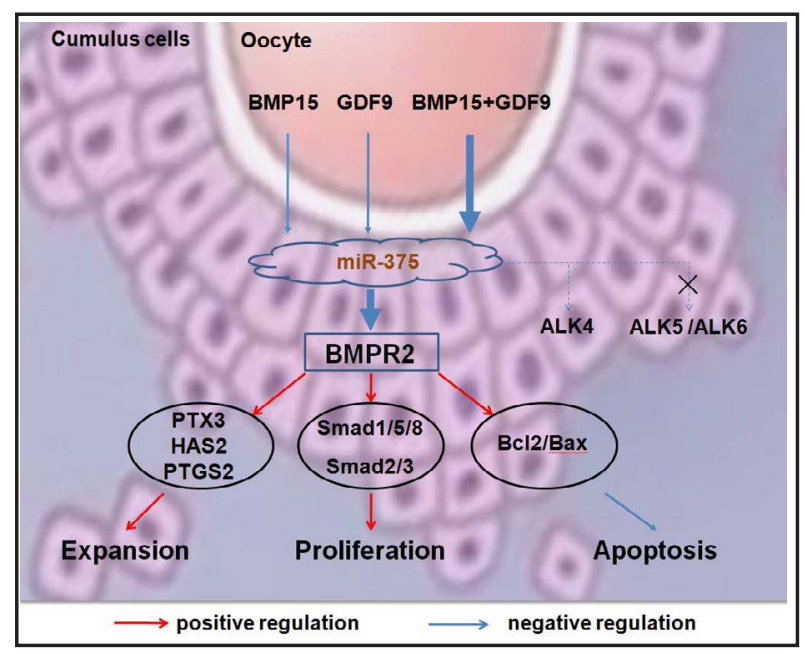

Fig. 8. The molecular mechanism of miR-375-BMPR2 on cumulus cell expansion, proliferation and apoptosis. The target gene for miR-375 is BMPR2, the only type II receptor of BMP15 and GDF9. Addition of BMP15 or GDF9 caused miR-375 down-regulation and BMPR2 up-regulation. MiR375 indirectly regulated the expression of ALK4 but had no effect on ALK5/6. Second, BMPR2 had no significant effect on the level of Smad1/5/8 or Smad2/3 but had varying degrees of influence on $\mathrm{p}-\mathrm{Smad} 1 / 5 / 8$ and $\mathrm{p}-\mathrm{Smad} 2 / 3$. These effects were more pronounced with the combination of BMP15 and GDF9. We also found that over-expression of miR-375 (or inhibition of its target gene BMPR2) reduced the Bcl2: Bax ratio, downregulated the PTGS2, PTX3, and HAS2 genes, and increased the proportion of cumulus cell apoptosis. Therefore, we believe that miR-375 plays an important role in the biological process of oocyte regulation of cumulus cell proliferation and apoptosis through BMP15 and GDF9. 


\section{Cellular Physiology Cell Physiol Biochem 2018;46:213-225 \begin{tabular}{ll|l} 
and Biochemistry & $\begin{array}{l}\text { DOI: 10.1159/000488424 } \\
\text { Published online: March 26, } 2018\end{array}$ & $\begin{array}{l}\text { (c) } 2018 \text { The Author(s). Published by S. Karger AG, Basel } \\
\text { www.karger.com/cpb }\end{array}$
\end{tabular} \\ Liu et al.: BMP15/GDF9 Regulate Smad1/5/8 and Smad2/3 Signaling through MiR-375- BMPR2}

Our research group will also use high-throughput sequencing technology to explore more factors regulated by BMP15 and GDF9 in future studies and conduct in-depth analysis of the regulation and mechanisms by which BMP15 and GDF9 act on oocytes and the surrounding cumulus cells and granulosa cells.

\section{Acknowledgements}

This study was supported by the National Natural Science Foundation of China (31572400 and 31601940), the Modern Agricultural Industry Technology System (CARS37), the Science and Technology Project of Jilin Province (20160520049JH, 20160203014NY and 20170203010NY), and the Technical System of Beef Cattle in Jilin Province (201635).

\section{Disclosure Statement}

No conflict of interests exists.

\section{References}

1 Glister C, Kemp CF, Knight PG: Bone morphogenetic protein (BMP) ligands and receptors in bovine ovarian follicle cells: actions of BMP-4, -6 and -7 on granulosa cells and differential modulation of Smad-1 phosphorylation by follistatin. Reproduction 2004;127:239-254.

-2 Su YQ, Wu X, O’Brien MJ, Pendola FL, Denegre JN, Matzuk MM, Eppig JJ: Synergistic roles of BMP15 and GDF9 in the development and function of the oocyte-cumulus cell complex in mice: genetic evidence for an oocyte-granulosa cell regulatory loop. Dev Biol 2004;276:64-73.

-3 Yan C, Wang P, DeMayo J, DeMayo FJ, Elvin JA, Carino C, Prasad SV, Skinner SS, Dunbar BS, Dube JL, Celeste AJ, Matzuk MM: Synergistic roles of bone morphogenetic protein 15 and growth differentiation factor 9 in ovarian function. Mol Endocrinol 2001;15:854-866.

-4 Yoon JD, Jeon Y, Cai L, Hwang SU, Kim E, Lee E, Kim DY, Hyun SH: Effects of coculture with cumulus-derived somatic cells on in vitro maturation of porcine oocytes. Theriogenology 2015;83:294-305.

5 Abir R, Fisch B, Johnson MH: BMP15, fertility and the ovary. Reprod Biomed Online 2014;29:525-526.

6 Caixeta ES, Sutton-McDowall ML, Gilchrist RB, Thompson JG, Price CA, Machado MF, Lima PF, Buratini J: Bone morphogenetic protein 15 and fibroblast growth factor 10 enhance cumulus expansion, glucose uptake, and expression of genes in the ovulatory cascade during in vitro maturation of bovine cumulusoocyte complexes. Reproduction 2013;146:27-35.

7 Su YQ, Sugiura K, Wigglesworth K, O’Brien MJ, Affourtit JP, Pangas SA, Matzuk MM, Eppig JJ: Oocyte regulation of metabolic cooperativity between mouse cumulus cells and oocytes: BMP15 and GDF9 control cholesterol biosynthesis in cumulus cells. Development 2008;135:111-121.

8 Zhai B, Liu H, Li X, Dai L, Gao Y, Li C, Zhang L, Ding Y, Yu X, Zhang J: BMP15 prevents cumulus cell apoptosis through CCL2 and FBN1 in porcine ovaries. Cell Physiol Biochem 2013;32:264-278.

9 Pangas SA, Matzuk MM: The art and artifact of GDF9 activity: cumulus expansion and the cumulus expansion-enabling factor. Biol Reprod 2005;73:582-585.

10 Shi FT, Cheung AP, Huang HF, Leung PC: Growth differentiation factor 9 (GDF9) suppresses follistatin and follistatin-like 3 production in human granulosa-lutein cells. PLoS One 2011;6:e22866.

$\rightarrow 11$ Tuck AR, Mottershead DG, Fernandes HA, Norman RJ, Tilley WD, Robker RL, Hickey TE: Mouse GDF9 decreases KITL gene expression in human granulosa cells. Endocrine 2015;48:686-695.

12 van Caam A, Madej W, Garcia de Vinuesa A, Goumans MJ, Ten Dijke P, Blaney Davidson E, van der Kraan P: TGFbeta1-induced SMAD2/3 and SMAD1/5 phosphorylation are both ALK5-kinase-dependent in primary chondrocytes and mediated by TAK1 kinase activity. Arthritis Res Ther 2017;19:112.

13 Gilchrist RB, Lane M, Thompson JG: Oocyte-secreted factors: regulators of cumulus cell function and oocyte quality. Hum Reprod Update 2008;14:159-177. 


\section{Cellular Physiology Cell Physiol Biochem 2018;46:213-225 \begin{tabular}{l|l|l}
\hline DOI: 10.1159/000488424 & $\begin{array}{l}\text { C) 2018 The Author(s). Published by S. Karger AG, Basel } \\
\text { www.karger.com/cpb }\end{array}$
\end{tabular}}

Liu et al.: BMP15/GDF9 Regulate Smad1/5/8 and Smad2/3 Signaling through MiR-375BMPR2

14 Fenwick MA, Mora JM, Mansour YT, Baithun C, Franks S, Hardy K: Investigations of TGF-beta signaling in preantral follicles of female mice reveal differential roles for bone morphogenetic protein 15 Endocrinology 2013;154:3423-3436.

15 Bradshaw RA, Dennis EA: Handbook of cell signaling, Academic Press, Amsterdam San Diego, Calif., 2004.

-16 Hussein TS, Froiland DA, Amato F, Thompson JG, Gilchrist RB: Oocytes prevent cumulus cell apoptosis by maintaining a morphogenic paracrine gradient of bone morphogenetic proteins. J Cell Sci 2005;118:52575268.

17 Kang H, Louie J, Weisman A, Sheu-Gruttadauria J, Davis-Dusenbery BN, Lagna G, Hata A: Inhibition of microRNA-302 (miR-302) by bone morphogenetic protein 4 (BMP4) facilitates the BMP signaling pathway. J Biol Chem 2012;287:38656-38664.

18 Larabee SM, Coia H, Jones S, Cheung E, Gallicano GI: miRNA-17 members that target Bmpr2 influence signaling mechanisms important for embryonic stem cell differentiation in vitro and gastrulation in embryos. Stem Cells Dev 2015;24:354-371.

19 Qin W, Zhao B, Shi Y, Yao C, Jin L, Jin Y: BMPRII is a direct target of miR-21 Acta Biochim Biophys Sin (Shanghai) 2009;41:618-623.

-20 Zeng Y, Qu X, Li H, Huang S, Wang S, Xu Q, Lin R, Han Q, Li J, Zhao RC: MicroRNA-100 regulates osteogenic differentiation of human adipose-derived mesenchymal stem cells by targeting BMPR2. FEBS Lett 2012;586:2375-2381.

21 Li Y, Wang J, Zhang Z, Yi J, He C, Wang F, Tian X, Yang M, Song Y, He P, Liu G: Resveratrol compares with melatonin in improving in vitro porcine oocyte maturation under heat stress. J Anim Sci Biotechnol 2016;7:33.

22 Huang Z, Wells D: The human oocyte and cumulus cells relationship: new insights from the cumulus cell transcriptome. Mol Hum Reprod 2010;16:715-725.

-23 Yuan B, Sun GJ, Zhang GL, Wu J, Xu C, Dai LS, Chen J, Yu XF, Zhao ZH, Zhang JB: Identification of target genes for adenohypophysis-prefer miR-7 and miR-375 in cattle. Genet Mol Res 2015;14:9753-9763.

-24 Wu Y, Sun X, Song B, Qiu X, Zhao J: MiR-375/SLC7A11 axis regulates oral squamous cell carcinoma proliferation and invasion. Cancer Med 2017;6:1686-1697.

25 Chen W, Woodruff TK, Mayo KE: Activin A-induced HepG2 liver cell apoptosis: involvement of activin receptors and smad proteins. Endocrinology 2000;141:1263-1272.

-26 Hashimoto O, Yamato K, Koseki T, Ohguchi M, Ishisaki A, Shoji H, Nakamura T, Hayashi Y, Sugino H, Nishihara T: The role of activin type I receptors in activin A-induced growth arrest and apoptosis in mouse B-cell hybridoma cells. Cell Signal 1998;10:743-749.

-27 Kristensen SG, Andersen K, Clement CA, Franks S, Hardy K, Andersen CY: Expression of TGF-beta superfamily growth factors, their receptors, the associated SMADs and antagonists in five isolated sizematched populations of pre-antral follicles from normal human ovaries. Mol Hum Reprod 2014;20:293308.

28 Mottershead DG, Sugimura S, Al-Musawi SL, Li JJ, Richani D, White MA, Martin GA, Trotta AP, Ritter LJ, Shi J, Mueller TD, Harrison CA, Gilchrist RB: Cumulin, an Oocyte-secreted Heterodimer of the Transforming Growth Factor-beta Family, Is a Potent Activator of Granulosa Cells and Improves Oocyte Quality. J Biol Chem 2015;290:24007-24020.

29 Jang YJ, Park JI, Moon WJ, Dam PT, Cho MK, Chun SY: Cumulus cell-expressed type I interferons induce cumulus expansion in mice. Biol Reprod 2015;92:20.

-30 Liang S, Guo J, Jin YX, Yuan B, Zhang JB, Kim NH: C-Phycocyanin supplementation during in vitro maturation enhances pre-implantation developmental competence of parthenogenetic and cloned embryos in pigs. Theriogenology 2018;106:69-78. 